\title{
Ab-initio calculations on static and dynamic third-order optical nonlinearity of azo-azulenes
}

\author{
A. Karakas ${ }^{1}$, Y. El kouari ${ }^{2}$, A. Migalska- Zalas ${ }^{3}$ and B. Sahraoui ${ }^{4}$ \\ ${ }^{I}$ Selcuk University, Faculty of Sciences, Department of Physics, Campus, Konya, Turkey; \\ ${ }^{2}$ Département Génie Electrique, Faculté des Sciences et Techniques, \\ Université Hassan II, B.P. 146, Mohammédia, 20650 Maroc; \\ ${ }^{3}$ Institute of Physics, J. Dlugosz Academy of Czestochowa PL-42217, Aleja Armii Krajowej \\ 13/15, Czestochowa, Poland; \\ ${ }^{4}$ LUNAM Université, Université d'Angers, CNRS UMR 6200, Laboratoire MOLTECH-Anjou, \\ 2 bd Lavoisier, 49045 ANGERS, France.
}

Received March 19, 2012; accepted March 28, 2012; published March 31, 2012

\begin{abstract}
To provide an insight into the microscopic third-order nonlinear optical (NLO) behavior of a series of azo-azulenes; both dispersion-free (static) and also frequency-dependent (dynamic) linear polarizabilities $(\alpha)$ and second hyperpolarizabilities $(\gamma)$ at $532 \mathrm{~nm}$ wavelength have been theoretically investigated by means of a timedependent Hartree-Fock (TDHF) method. The maximum one-photon absorption (OPA) wavelengths recorded by quantum mechanical computations using a configuration interaction (CI) method are estimated in the visible region, supporting the $\pi \rightarrow \pi^{*}$ transitions. The ab-initio calculation results with non-zero values on (hyper)polarizabilities indicate that the title compounds might possess microscopic third-order NLO phenomena.
\end{abstract}

NLO materials have been extensively studied for many years [1]. The search of new materials with NLO properties is an important research field [2]. A significant interest still exists in the design and development of materials exhibiting a large second-order NLO response because of the potential application in telecommunications, optical computing and optical signal processing [3]. Actually, a third-order response governed by the second hyperpolarizability offers more varied and richer behavior than a second-order NLO process due to the higher dimensionality of the frequency space. Due to potential applications in various photonic technologies, the NLO properties of organic molecular materials have been the object of intense research [4]. Devices for applications in optical communications, optical switches and wavelength filters have been created using the NLO response properties of organic systems. Therefore, organic molecules have been intensively studied with respect to their potential applications as NLO media [5].

The azo-azulene derivatives are interesting materials for the study of second and third-order NLO properties [6]. The azulenes possess both electron donating and electron accepting character through a five-membered ring and a seven-membered unsaturated ring, respectively. Connecting the donor and acceptor moieties with a conjugated bridge is a well established strategy to obtain the molecules with high-order hyperpolarizability tensors. Based on the structure and previous studies, one could expect that the title azo-azulene derivatives (Fig. 1) may show third-order NLO behavior. The aim of this paper is to determine the third-order optical nonlinearities of the examined compounds by the theoretical approaches. So, we present here an ab-initio study utilizing the TDHF procedure on disperison-free and frequency-dependent linear polarizabilities and second hyperpolarizabilities.
AHP<smiles></smiles>

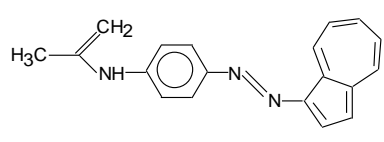

AAP
ABT
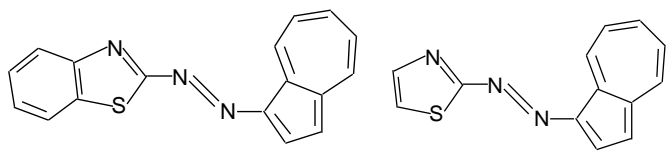

AT

Fig. 1. Structures of the studied compounds AHP - azulene-1-azo-(4hydroxyphenyl) AAP - azulene-1-azo-(4-acetamidophenyl) ABT - azulene-1-azo-(2-benzothiazole) AT - azulene-1-azo-(2thiazole).

The theoretical computations involve the determination of dispersion-free and frequency-dependent linear polarizability and second hyperpolarizability tensor components of AAP, ABT, AHP, AT. The molecule geometries have been firstly optimized on the ab-initio restricted closed-shell Hartree-Fock level. The optimized structures have been used to compute the linear polarizabilities and third-order hyperpolarizabilities with $6-311+\mathrm{G}(d, p)$ polarized and diffused basis set. According to Bartlett et al. [7] very large basis sets are extremely important in accurate predictions of static hyperpolarizability. To avoid the problem of basis set completeness, various basis sets (including polarized and diffuse functions) have been implemented by Kenawi et 
al. [8], at the Hartree-Fock level of theory, to calculate the (hyper)polarizabilities of diclofenac sodium. The results obtained [8] show the importance of including the $p$ and $d$ polarized functions in the double split valence set for the best description of the static dipole (hyper)polarizabilities of diclofenac sodium. Small molecules require quite extended basis sets [9] because intra-atomic polarization is important and its accurate calculation needs a more precise choice of basis sets, especially when it has diffuse functions which would be almost similar to describing an isolated atom. On the other hand, inter-atomic valence polarization might not be as demanding in all cases; it essentially depends on the size and geometric orientation of the molecule. However, when exploring the accurate determination of dipole hyperpolarizabilities, both types of valence polarization require a careful choice of basis sets as reported by Maroulis [10], who utilized a systematic sequence of carefully optimized basis sets to calculate the static dipole hyperpolarizability of transbutadiene. One expects the basis set $6-311+\mathrm{G}(d, p)$ to yield molecular property values of near-Hartree-Fock quality. The ab-initio TDHF method is one of the most useful among the computational procedures to calculate (hyper)polarizabilities [4]. $\alpha(0 ; 0)$ and $\gamma(0 ; 0,0,0)$ at $\omega=0$; $\alpha(-\omega ; \omega)$ and $\gamma(-3 \omega ; \omega, \omega, \omega)$ calculations at $\omega=0.08562$ atomic units (a. u.) (i.e., at $\lambda=532 \mathrm{~nm}$ wavelength), often used laser frequency in third-harmonic generation (THG) measurements, have been carried out using the TDHF method implemented in the GAMESS [11] program. In these $\gamma$ definitions above mentioned, the first describes static third-order hyperpolarizabilities, the second represents the hyperpolarizability for frequency tripling, called a third-harmonic generation (THG) process.

In this study, the average linear polarizability $\langle\alpha>$ and third-order hyperpolarizability $\langle\gamma\rangle$ values have been calculated using the following expressions, respectively [12]:

$$
\begin{aligned}
& <\alpha>=\left(\alpha_{\mathrm{xx}}+\alpha_{\mathrm{yy}}+\alpha_{\mathrm{zz}}\right) / 3 \\
& <\gamma>=\left[\gamma_{\mathrm{xxxx}}+\gamma_{\mathrm{yyyy}}+\gamma_{\mathrm{zzzz}}+2\left(\gamma_{\mathrm{xxyy}}+\gamma_{\mathrm{xxzz}}+\gamma_{\mathrm{yyzz}}\right)\right] / 5
\end{aligned}
$$

To calculate all the (hyper)polarizabilities, the origins of the Cartesian coordinate system $(x, y, z)=(0,0,0)$ have been chosen at the centers of mass of AAP, ABT, AHP, AT. All static and dynamic (hyper)polarizability calculations have been performed on a PC with an Intel (R) core (TM) I7-2630QM operator, 5.8GB RAM memory and $2 \mathrm{GHz}$ frequency using Linux PC GAMESS version running under Linux Fedora release 11 (Leonidas) environment.

Besides, the $\pi \rightarrow \pi^{*}$ transition wavelengths $\left(\lambda_{\max }\right)$ of the lowest lying electronic transition for AAP, ABT, AHP, AT have been studied theoretically by the electron excitation configuration interaction using the CIS/6-31G method in the GAUSSIAN03W [13] program.
NLO techniques are considered as among the most structure-sensitive methods to study molecular structures and assemblies [14]. Quantum chemistry calculations have been shown to be useful in the description of the relationship among the electronic structure of the systems and their NLO response. Actually, to accurately compute NLO properties of rather great molecular systems, there are well-tested computational codes. In particular, an accurate analysis of the NLO behavior of those great molecules leads to the definition of high-order hyperpolarizability values as well as third-order. Once conceived, the idea can be first pursued by theoretical means, and promising results would justify experimental efforts to obtain the envisioned compounds. The wavelengths obtained by UV-Vis spectral analysis can be helpful in planning the synthesis of promising NLO materials [15]. Since it is necessary to know the transparency region, the electronic absorption spectral studies of compounds designed to possess NLO properties are important. Albert et al. [16] have reached the conclusion that with the correct substitution of the pushpull system in the porphyrin ring, characterized by strong intramolecular $\pi \rightarrow \pi^{*}$ charge transfer transitions found through UV-Vis spectral analysis, some specific electronic and structural properties of this system could produce high NLO responses. Di Bella et al. [17] have reported that bis(salicyl-aldiminato)nickel(II) compound exhibited interesting linear optical features which will be seen to be related to the NLO response. A broad band has been found in the region between 300 and $360 \mathrm{~nm}$ involving mainly $\pi \rightarrow \pi^{*}$ transitions [17]. In this paper, vertical transition energies from the ground state to each excited state have been computed, giving OPA, i.e. the UV-Vis spectrum. The calculated wavelengths $\left(\lambda_{\max }\right)$ for the maximum OPA of the investigated molecules are shown in Table 1. $\lambda_{\max }$ values located in the visible region are very similar and may support the assignments of observed maxima to $\pi \rightarrow \pi *$ transitions.

Table 1. The maximum OPA wavelengths for AHP, AAP, ABT, AT.

\begin{tabular}{ccccc}
\hline Compound & AHP & AAP & ABT & AT \\
$\lambda_{\max }[\mathrm{nm}]$ & 496.77 & 494.60 & 480.51 & 473.33 \\
\hline
\end{tabular}

For more precise determination of the second hyperpolarizabilities an extended basis set is required [18]. The 6-311+G $(d, p)$ basis set utilized here provides a reliable computational tool for the study of third-order optical nonlinearity, and is probably rather adequate to compute the (hyper)polarizabilities of the title molecules. One could determine the hyperpolarizability tensors of molecules using a suitable computational approach. These tensors describe the response of molecules to an external electric field. In spite of the rapid development of highly advanced experimental techniques on NLO, the theoretical understanding of the NLO properties show that 
the responses of the molecule to the external application of an electric field are also very important. Because the TDHF is rather useful among the various computational procedures specified in Ref. [4], to calculate the NLO properties of azo-azulenes (Fig. 1) we have preferred using the TDHF method. In our theoretical calculations, the TDHF method has been found to have, on the one hand, high efficiency and appropriateness between this level of theory employed and the accuracy of calculations performed here and, on the other hand, computational capabilities. Hartree-Fock values are true reference results. It is rather difficult to analyze the performance of high-level theoretical studies in the absence of such references [19]. Our approach to the calculation of (hyper)polarizabilities relies on the TDHF method. Some significant calculated magnitudes of static and frequencydependent linear polarizabilities and second hyperpolarizabilities are shown in Tables 2-5, respectively. This kind of molecules has third-order nonlinearity with non-zero hyperpolarizabilities since the radicals increase their NLO effects, and thus intensify the asymmetric distortion of the conjugated system. Furthermore, the electronic properties of these molecules exhibit an unusual property, characterizing the intramolecular charge transfer between the two radicals [20]. We have found rather similar average dipole polarizabilities for all the investigated compounds (see Tables 2-3). However, the dynamic $\langle\gamma\rangle$ value of AT with thiazole-based has been calculated to be larger than the other azo-azulenes in Fig. 1 (Table 5). All studied compounds may have miscroscopic cubic nonlinearity with non-zero (hyper)polarizabilities (Tables 2-5).

Table 2. Some selected components of the static $\alpha(0 ; 0)$ and $<\alpha>(0 ; 0)$ $\left(* 10^{-24}\right.$ esu) values for AHP, AAP, ABT, AT.

\begin{tabular}{lcccc}
\hline compound & $\alpha_{\mathrm{xx}}$ & $\alpha_{\mathrm{yy}}$ & $\alpha_{\mathrm{zz}}$ & $<\alpha>$ \\
\hline AHP & 23.853 & 3.001 & 43.444 & 23.433 \\
AAP & 26.438 & 5.621 & 50.134 & 27.398 \\
ABT & 26.625 & 4.454 & 52.744 & 27.941 \\
AT & 22.254 & 3.583 & 39.141 & 21.659 \\
\hline
\end{tabular}

Table 3. Some selected components of the frequency-dependent $\alpha(-\omega ; \omega)$ and $\left\langle\alpha>(-\omega ; \omega) \quad\left(* 10^{-24}\right.\right.$ esu $)$ values at $\omega=0.08562$ a.u. for AHP, AAP, ABT, AT.

\begin{tabular}{lcccc}
\hline compound & $\alpha_{\mathrm{xx}}$ & $\alpha_{\mathrm{yy}}$ & $\alpha_{\mathrm{zz}}$ & $<\alpha>$ \\
\hline AHP & 39.472 & 25.622 & 2.758 & 22.618 \\
AAP & 44.111 & 28.436 & 5.556 & 26.034 \\
ABT & 48.674 & 28.005 & 3.501 & 26.727 \\
AT & 36.887 & 23.391 & 2.829 & 21.036 \\
\hline
\end{tabular}

Table 4. All static $\gamma(0 ; 0,0,0)$ components and $\langle\gamma\rangle(0 ; 0,0,0)\left(* 10^{-37}\right.$ esu $)$ values for AHP, AAP, ABT, AT.

\begin{tabular}{lccccccc}
\hline & $\gamma_{\mathrm{xxxx}}$ & $\gamma_{\mathrm{yyyy}}$ & $\gamma_{\mathrm{zzzz}}$ & $\gamma_{\mathrm{xxyy}}$ & $\gamma_{\mathrm{xxzz}}$ & $\gamma_{\mathrm{yyzz}}$ & $\langle\gamma\rangle$ \\
\hline AHP & 1154.498 & -11.958 & -0.173 & -10.618 & 3.628 & 1.617 & 226.323 \\
AAP & 1379.315 & -20.273 & -0.123 & 17.637 & 5.131 & 1.581 & 281.523 \\
ABT & 1896.415 & -10.107 & -0.082 & -18.015 & 5.984 & 2.288 & 373.348 \\
AT & 1097.355 & -5.574 & -0.153 & -26.420 & 3.037 & 1.985 & 209.766 \\
\hline
\end{tabular}

Table 5. Some selected components of the frequency-dependent $\gamma(-3 \omega$; $\omega, \omega, \omega)$ and $\langle\gamma\rangle(-3 \omega ; \omega, \omega, \omega)\left(* 10^{-37} \mathrm{esu}\right)$ values at $\omega=0.08562$ a.u. for AHP, AAP, ABT, AT.

\begin{tabular}{cccccccc}
\hline & $\gamma_{\mathrm{xxxx}}$ & $\gamma_{\mathrm{yyyy}}$ & $\gamma_{z z z z}$ & $\gamma_{\mathrm{xxyy}}$ & $\gamma_{\mathrm{xxzz}}$ & $\gamma_{\mathrm{yyzz}}$ & $\langle\gamma\rangle$ \\
\hline AHP & -4315.055 & -2509.046 & -0.259 & -291.360 & -1.327 & 16.302 & -1353.923 \\
AAP & -3572.722 & 1235.451 & 0.178 & -71.205 & -0.539 & -13.226 & -891.172 \\
ABT & 11437.268 & 545.530 & 109.727 & -1186.566 & 59.735 & -14.296 & 1323.872 \\
AT & 12737.343 & -3127.622 & 0.364 & -1457.532 & 14.266 & 27.291 & 1777.178 \\
\hline
\end{tabular}

In conclusion, we have presented the results of computational studies, showing how the title compounds can possess third-order optical nonlinearity. To test the microscopic third-order NLO behavior the computations of OPA wavelengths, linear and second (hyper)polarizabilities may be considered quite adequate. According to the results of the calculation on linear optical behavior, electronic transition wavelengths are estimated in the visible region assignable to the $\pi \rightarrow \pi^{*}$ transitions. The ab-initio computed non-zero (hyper)polarizability values imply that the examined azoazulenes might have microscopic third-order NLO phenomena. It is seen from Table 5 that compound AT with thiazole fragment induces the strongest cubic nonlinearity of a family of azo-azulenes in Fig. 1. The comparison of our theoretical results with experimental data will be the subject of a separate work.

\section{References}

[1] D.M. Burland, R.D. Miller, C.A. Walsh, Chem. Rev. 94, 31 (1994).

[2] T.G. Pedersen, K. Pedersen, P. K. Kristensen, J. Rafaelsen, N. Skivesen, Z. Li, S. V. Hoffmann, Surf. Sci. 516, 127 (2002).

[3] A. Karakaş, Z. Erdem Koç, M. Fridrichová, P. Němec, J. Kroupa, J. Theoretical and Computational Chemistry 11, 209 (2012).

[4] D.R. Kanis, M.A. Ratner, T. Marks, Chem. Rev. 94, 195 (1994).

[5] A. Elmali, A. Karakaş, H. Ünver, Chem. Phys. 309, 251 (2005).

[6] Z. Essaidi, J. Nizioł, B. Sahraoui, Opt. Materials 33(9), 1387 (2011).

[7] R.J. Bartlett, H. Sekino, G.D. Purvis, Chem. Phys. Lett. 98, 66 (1983).

[8] I.M. Kenawi, A.H. Kamel, R.H. Hilal, J. Mol. Struct. 851, 46 (2008).

[9] C.E. Dykstra, J. Chem. Phys. 82, 4120 (1985)

[10] G. Maroulis, J. Chem. Phys. 111, 583 (1999).

[11] Intel $\times 86$ (win32, Linux, OS/2, DOS) version. PC GAMESS version 6.2, build number 2068; described in: M. W. Schmidt et. al., J. Comput. Chem. 14, 1347 (1993)

[12] M.P. Bogaard, B. Orr, Ed.: A.D. Buckingham, MTP International review of science, vol. 2 (Butterworths, London 1975).

[13] M.J. Frisch et al., Gaussian 03, Revision E.01 (Gaussian, Inc., Wallingford CT 2004).

[14] Y.R. Shen, Proc. Natl. Acad. Sci. U.S.A. 93, 112104 (1996).

[15] J. Kulakowska, S. Kucharski, Eur. Polym. J. 36, 1805 (2000)

[16] I.D.L. Albert, T.J. Marks, M. A. Ratner, Chem. Mater. 10, 753 (1998).

[17] S. Di Bella, I. Fragalà, I. Ledoux, M.A. Diaz-Garcia, P.G. Lacroix, T.J. Marks, Chem. Mater. 6, 881 (1994).

[18] J.L. Brédas, C. Adant, P. Tackx, A. Persoons, Chem. Rev. 94, 243 (1994).

[19] G. Maroulis, D. Xenides, U. Hohm, A. Loose, J. Chem. Phys. 115, 7957 (2001).

[20] G. Kelly, F. Darviche, N. Robertson, T. Gelbrich, M. B. Hursthouse, D.A. Thomas, I. R. Butler, Inorganic Chem. Com. 8 (No. 10), 874 (2005). 